\title{
Evaluation of the botanical origin of commercial dry bee pollen load batches using pollen analysis: a proposal for technical standardization
}

\author{
ORTRUD M. BARTH ${ }^{1,2}$, ALEX S. FREITAS ${ }^{1}$, ÉRIKA S. OLIVEIRA ${ }^{1}$, ROSANA A. SILVA ${ }^{3}$, \\ FERNANDA M. MAESTER ${ }^{3}$, RAQUEL R.S. ANDRELLA ${ }^{3}$ and GINA M.B.Q. CARDOZO ${ }^{3}$ \\ ${ }^{1}$ Laboratório de Palinologia, Departamento de Botânica, Instituto de Biologia, Universidade Federal do Rio de Janeiro, \\ Ilha do Fundão, Centro de Ciências da Saúde, Bloco A, Sala 118, 21949-900 Rio de Janeiro, RJ, Brasil \\ ${ }^{2}$ Instituto Oswaldo Cruz, Fundação Oswaldo Cruz, Avenida Brasil 4365, 21040-900 Rio de Janeiro, RJ, Brasil \\ ${ }^{3}$ ITAL - Instituto de Tecnologia de Alimentos, Centro de Ciência e Qualidade de Alimentos, \\ Avenida Brasil 2880, 13070-178 Campinas, SP, Brasil
}

Manuscript received on August 20, 2009; accepted for publication on April 8, 2010

\begin{abstract}
High quality of bee pollen for commercial purpose is required. In order to attend the consumer with the best identification of the botanical and floral origin of the product, 25 bee pollen batches were investigated using two techniques of pollen grain preparation. The first started to identify pollen loads of different colors in two grams of each well mixed batch, and the second to identify pollen grains in a pool made of all the pollen loads comprised in two grams. The best result was obtained by this last technique, when a pollen grain suspension was dropped on a microscope slide and circa 500 pollen grains were counted per sample. This analysis resulted in the recognition of monofloral and bifloral pollen batches, while the use of the first technique resulted in all samples receiving a heterofloral diagnosis.
\end{abstract}

Key words: Apis, pollen loads, commercial pollen batches, pollen analysis, botanical origin.

\section{INTRODUCTION}

Bee pollen production increased during the last years as a response to commercial demand. Apis mellifera $\mathrm{L}$. is the best pollen supplier mainly in tropical countries where entomophilous plant species are dominant. Beekeepers use pollen traps of different types in order to obtain the pollen loads from bees when coming home to hives. This pollen is humid and has to be dried before commercialization. Then, it may be distributed in vials receiving an identification that comprises, in addition, its botanical origin. This procedure is important to avoid missing the accurate scientific definition of the botanical name, since beekeepers report a lot of common names of plants that were visited by the bees.

Pollen grains present a huge variation of morphological features that are established by its genetic hered-

Correspondence to: Ortrud Monika Barth

E-mail: barth@ioc.fiocruz.br ity and are not influenced by environmental events or changes. Pollen analysis is the tool to recognize from where the pollen grains are coming. Several laboratorial techniques are used to prepare pollen grains for microscope observation and identification. The difficulty remains in the interpretation of the obtained data and the evaluation of numerical and personal informations. Several aspects are to be considered, the first one regarding the bee behavior upon flower visitation for pollen extraction. In this case, single bees are captured and both of their pollen loads were analyzed (Carvalho and Marchini 1999, Marques-Souza et al. 2002, Noor et al. 2009). Secondly, when pollen traps are used, several pollen loads perform one sample that, for analysis, has to be well mixed; a group of pollen loads, in general selected by its weight or color (Almeida-Muradian 2005, Barth et al. 2009, Modro et al. 2009a), is used for the recognition of the botanical species composing this pollen sample. 
The main issue to address is the significance or not of the choice by color. An accurate description of the pollen load sample processing and analysis is frequently missed during experiment descriptions (Modro et al. 2007, 2009a). Commonly, a fragment of a pollen load of each color is analyzed; no sample centrifugation is used. In another way (denoted technique 1), ten pollen loads (Barth et al. 2009) or 25 ones of each color batch (Barth, personal information and in the present paper) are mixed using ethanol, and a little amount is analyzed after centrifugation. As pollen grains have different weight, a gradient is obtained, from which pollen grains are removed using a piece of glycerin jelly. The significance of the obtained results is not clear. Another technically very complex preparation of pollen batches identification was recently used comprising several steps of pollen drying, hydrating and acetolysis (Novais et al. 2009) and, thus, missing important information besides pollen morphology.

The proposed technique by this study (technique 2) may help to get quick and accurate information about the botanical and regional origin of commercial bee pollen load products. It neither demands long time to be performed nor is expensive.

\section{MATERIALS AND METHODS}

Twenty five samples of commercial batches of bee pollen loads were obtained in apiaries of several states in Brazil and analyzed (Table I). Each sample was analyzed using two techniques. The first technique considers sub samples according to the pollen load colors; each sample was made of well-mixed two grams and each sub sample was made of 25 units or pollen loads of each color. When two or more types of color were mentioned in a same sub sample (Table I, column 2), it indicates that no sufficient pollen loads of a unique color were available to perform $n=25$, and several poorly represented color batches were mixed. Color was determined according to the Red Green Blue (RGB) color classification system. The color values correspond to: $b r i g h t=$ RGB 247/240/183; brown $=$ RGB 186/97/97, caramel $=$ RGB 237/202/29, dark $=$ RGB 117/11/11, green $=$ RGB $98 / 156 / 128$, orange $=$ RGB $255 / 106 / 37$, violet $=$ RGB $123 / 102 / 147$, yellow $=$ RGB 250/224/23. The second technique used all pollen loads together comprised in two grams of a commercial batch sample.
Pollen grain identification used the available literature, meanly Barth (1989), Roubik and Moreno (1991), and the Palynological Slide Collection of the Laboratory of Palynology. Pollen frequency standard classes (Louveaux et al. 1978) were used, making an additional subdivision of the dominant pollen class (Table I).

Technique 1 (TABle I, Columns 2-4):

\section{Preparation of pollen load samples:}

The pollen loads of two grams of each sample were distributed in batches or sub samples according to its color.

\section{Preparation of microscope slides:}

Twenty five pollen loads of each sub sample were macerated using $10 \mathrm{~mL}$ of $70 \%$ ethanol distributed into two $15 \mathrm{~mL}$ centrifuge tubes and centrifuged during 3 minutes at $1500 \mathrm{rpm}$. The sediment was resuspended with $10 \mathrm{~mL}$ of $70 \%$ ethanol and centrifuged again. In sequence, $5 \mathrm{~mL}$ of a water/glycerin 1:1 mixture were added to each tube, stirred, and left for 30 minutes. After centrifugation, the tubes remained with their aperture down on absorbent paper during a few minutes. Then, the pollen sediment was mixed and pollen slides were prepared using a little piece of glycerin jelly to capture the pollen grains, and slides were sealed with paraffin. Counting and identification comprised at least 500 pollen grains.

\section{Technique 2 (TABle I, Columns 5-6):}

\section{Preparation of pollen load samples:}

Dry pollen loads of two grams of a sample (this is a pool of circa 300 pollen loads) were weighted into a $15 \mathrm{~mL}$ Falcon centrifuge tube, mixed using $70 \%$ ethanol just to complete $13 \mathrm{~mL}$, and left for 30 minutes. Treatment with ultrasound (if disposable) during 5 minutes can be suitable for particle dispersal. The sediment obtained after centrifugation may be extracted with ethanol, and submitted to ultrasonic treatment again. A solution of distilled water/glycerin 1:1 was added to the sediment just to complete $13 \mathrm{~mL}$, and left for circa 30 minutes.

\section{Preparation of microscope slides:}

One drop of the well-mixed pollen grain suspension was applied on a microscope slide, covered with a $22 \times 22 \mathrm{~mm}$ cover slide, and sealed with enamel. The slide may be 

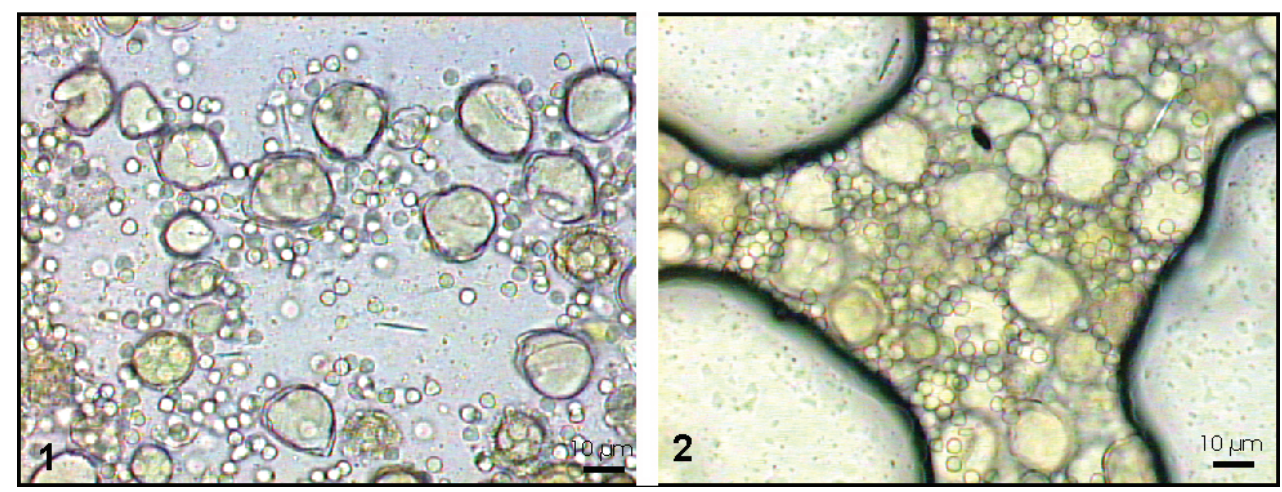

Fig. 1 - A pool of pollen loads prepared using technique 2; pollen grains are well dispersed all over the slide. Fig. 2 - A pool of pollen loads prepared using technique 2 three weeks later; the slide started to dry, and the pollen grains overlapped and were pressed against the air bubbles.

maintained in a horizontal position for circa one month or less, when drying starts, and the pollen grains were selectively pressed against the air bubbles. Then discard the slide (Figs. 1 and 2).

\section{Preparation of stock samples:}

One $\mathrm{mL}$ of the well mixed pollen grain suspension was put into a $1.5 \mathrm{~mL}$ Eppendorf centrifuge tube and centrifuged. A $15 \mathrm{~mL}$ Falcon tube can be used as a support for the Eppendorf tube. After discarding the supernatant, one $\mathrm{mL}$ of glycerin was added, mixed, and then the tube was closed and identified for additional preparations. It may be kept at room temperature.

\section{Preparation of additional pollen slides:}

The content of the Eppendorf tube has to be homogenized. One drop of this pollen grain suspension was mixed with one drop of distilled water in a clean Eppendorf tube. A microscope slide, as formerly described may be prepared after $30 \mathrm{~min}$ waiting for water impregnation.

\section{Counting and identification of pollen grains and structured elements:}

At least 500 pollen grains have to be counted and identified using a $400 \times$ magnification, preferentially within 10 days, before drying starts.

\section{RESULTS AND DISCUSSION}

The obtained data are shown in Table I. Each of the 25 samples analyzed presented two or more colors of pollen loads, establishing several sub samples. No sample was single colored. When no sufficient pollen loads of a unique color were available, two, rarely three different colored pollen loads were joined together in order to make up a group of 25 unities (column three); these never constituted a dominant sub group in a sample or batch.

Fifty two pollen types presenting a frequency equal or higher than $3 \%$ of the pollen sum were recognized. Technique 1 presented 46 pollen types, 22 being exclusives. Technique 2 presented 29 pollen types, six being exclusives.

No monofloral pollen load batch was recognized by technique 1 . Among the 25 analyzed samples, three were bifloral presenting two dominant pollen types; 22 samples were heterofloral comprising three or more pollen types. Considering technique 2, eight pollen load batches were monofloral, six bifloral and 11 heterofloral.

Nevertheless, technique 1 detected more pollen types (46) than technique 2 (29), this last procedure showed a better evaluation of the whole pollen batch. Thus, commercial qualification has to follow this way. Consequently, it presented more monofloral batches.

Technique 1 was useful in order to teach about pollen loads botanical origin. As explained below, the color of pollen of a plant species may vary (Barth et al. 2009 and in the present paper). Therefore, it is not a good characteristic to inform about the botanical origin of a pollen load batch. While it is not possible to analyze the pollen grain composition of each pollen load, and several plants may contribute to form a unique load, technique 2 is a quicker and more accurate procedure to make a diagnosis of a large commercial product. 
TABLE I

Comparison of 25 pollen load samples ( $2 \mathrm{~g}$ of each batch) from Apis mellifera evaluated by its color (sub samples in columns two, three and four) and evaluated by a pool of $2 \mathrm{~g}$ of each batch (columns five and six). Each sub sample comprised 25 units of pollen loads. Only the pollen types with frequency $>\mathbf{3} \%$ were considered: very frequent $(++++$, circa $>85 \%)$, frequent $(+++$, circa 45 to $85 \%)$, few frequent $(++$, circa 15 to $45 \%)$, rare $(+$, circa 3 to $15 \%)$.

\begin{tabular}{|c|c|c|c|c|c|}
\hline \multirow[b]{2}{*}{$\begin{array}{l}\text { Sample } \\
\text { register and } \\
\text { procedence }\end{array}$} & \multicolumn{3}{|c|}{ Technique 1} & \multicolumn{2}{|c|}{ Technique 2} \\
\hline & $\begin{array}{l}\text { Sub samples } \\
\text { established } \\
\text { by color of } \\
\text { pollen loads }\end{array}$ & $\begin{array}{l}\text { Pollen types } \\
\text { identified in the } \\
\text { sub samples }\end{array}$ & $\begin{array}{l}\text { Evaluation of } \\
\text { the sub samples }\end{array}$ & $\begin{array}{l}\text { Pollen types } \\
\text { identified in a pool } \\
\text { of } 2 \mathrm{~g} \text { of the sample }\end{array}$ & $\begin{array}{l}\text { Evaluation of } \\
\text { a pool of } 2 \mathrm{~g} \\
\text { of the sample }\end{array}$ \\
\hline \multirow[t]{4}{*}{ 55-Piaui } & yellow & Cyperus $(++++)$ & $\begin{array}{l}\text { monofloral of } \\
\text { Cyperus }\end{array}$ & \multirow{4}{*}{$\begin{array}{l}\text { Cocos nucifera }(+++) \\
\text { Cyperus }(++) \\
\text { Amaranthaceae }(+) \\
\text { Solanaceae }(+)\end{array}$} & \multirow{4}{*}{$\begin{array}{l}\text { bifloral of } \\
\text { Cocos nucifera } \\
\text { (dominant) } \\
\text { and Cyperus }\end{array}$} \\
\hline & $\begin{array}{l}\text { bright and } \\
\text { green }\end{array}$ & $\begin{array}{l}\text { Cocos nucifera } \\
(++++)\end{array}$ & $\begin{array}{l}\text { monofloral of } \\
\text { Cocos nucifera }\end{array}$ & & \\
\hline & dark & $\begin{array}{l}\text { Tapirira }(++++), \\
\text { Bombax }(+)\end{array}$ & $\begin{array}{l}\text { monofloral of } \\
\text { Tapirira }\end{array}$ & & \\
\hline & brown & $\begin{array}{l}\text { Inga }(++++), \\
\text { Cocos nucifera }(++)\end{array}$ & $\begin{array}{l}\text { monofloral of } \\
\text { Inga }\end{array}$ & & \\
\hline \multirow[t]{2}{*}{ 56-Bahia } & $\begin{array}{l}\text { bright and } \\
\text { orange }\end{array}$ & $\begin{array}{l}\text { Syagrus }(++++), \\
\text { Portulaca }(++)\end{array}$ & $\begin{array}{l}\text { monofloral of } \\
\text { Syagrus }\end{array}$ & \multirow[t]{2}{*}{$\begin{array}{l}\text { Cocos nucifera } \\
(++++)\end{array}$} & \multirow[t]{2}{*}{$\begin{array}{l}\text { monofloral of } \\
\text { Cocos nucifera }\end{array}$} \\
\hline & $\begin{array}{l}\text { bright and } \\
\text { green }\end{array}$ & $\begin{array}{l}\text { Mimosa scabrella } \\
(++++), \text { Fabaceae }(++)\end{array}$ & $\begin{array}{l}\text { monofloral of } \\
\text { Mimosa scabrella }\end{array}$ & & \\
\hline \multirow[t]{2}{*}{ 57-Bahia } & $\begin{array}{l}\text { bright and } \\
\text { orange }\end{array}$ & $\begin{array}{l}\text { Syagrus }(++++), \\
\text { Portulaca }(++)\end{array}$ & $\begin{array}{l}\text { monofloral of } \\
\text { Syagrus }\end{array}$ & \multirow{2}{*}{$\begin{array}{l}\text { Mimosa scabrela } \\
(++++) \\
\text { Syagrus }(+) \\
\text { Cocos nucifera }(+)\end{array}$} & \multirow{2}{*}{$\begin{array}{l}\text { monofloral of } \\
\text { Mimosa } \\
\text { scabrella }\end{array}$} \\
\hline & $\begin{array}{l}\text { brown and } \\
\text { green }\end{array}$ & $\begin{array}{l}\text { several polen } \\
\text { types }\end{array}$ & heterofloral & & \\
\hline \multirow[t]{3}{*}{ 58-Minas Gerais } & bright & $\begin{array}{l}\text { Brassica }(+++), \\
\text { Vernonia }(+)\end{array}$ & $\begin{array}{l}\text { monofloral of } \\
\text { Brassica }\end{array}$ & \multirow{3}{*}{$\begin{array}{l}\text { Eucalyptus }(+++) \\
\text { Cecropia }(++) \\
\text { Myrcia }(+) \\
\text { Syagrus }(+)\end{array}$} & \multirow{3}{*}{$\begin{array}{l}\text { heterofloral } \\
\text { with dominance } \\
\text { of Eucalyptus }\end{array}$} \\
\hline & orange & Senecio $(++++)$ & $\begin{array}{l}\text { monofloral of } \\
\text { Senecio }\end{array}$ & & \\
\hline & dark & $\begin{array}{l}\text { Vernonia }(++++) \\
\text { and uredospores }\end{array}$ & $\begin{array}{l}\text { monofloral of } \\
\text { Vernonia and } \\
\text { uredospores }\end{array}$ & & \\
\hline \multirow[t]{4}{*}{ 59-São Paulo } & caramel & $\begin{array}{l}\text { Poaceae }(+++) \\
\text { Anadenanthera }(+)\end{array}$ & $\begin{array}{l}\text { monofloral of } \\
\text { Poaceae }\end{array}$ & \multirow{4}{*}{$\begin{array}{l}\text { Eucalyptus }(+++) \\
\text { Vernonia }(++) \\
\text { Senecio }(++) \\
\text { Piper }(+) \\
\text { Myrcia }(+)\end{array}$} & \multirow[t]{4}{*}{ heterofloral } \\
\hline & bright & Syagrus $(++++)$ & $\begin{array}{l}\text { monofloral of } \\
\text { Syagrus }\end{array}$ & & \\
\hline & $\begin{array}{l}\text { dark and } \\
\text { yellow }\end{array}$ & Eucalyptus $(++++)$ & $\begin{array}{l}\text { monofloral of } \\
\text { Eucalyptus }\end{array}$ & & \\
\hline & orange & $\begin{array}{l}\text { without pollen } \\
\text { grains, only } \\
\text { uredospores }\end{array}$ & $\begin{array}{l}\text { without pollen } \\
\text { grains, only } \\
\text { uredospores }\end{array}$ & & \\
\hline \multirow[t]{3}{*}{ 65-Bahia } & $\begin{array}{l}\text { yellow and } \\
\text { brown }\end{array}$ & Cyperus $(++++)$ & $\begin{array}{l}\text { monofloral of } \\
\text { Cyperus }\end{array}$ & \multirow[t]{3}{*}{$\begin{array}{l}\text { Mimosa scabrella } \\
(++++)\end{array}$} & \multirow{3}{*}{$\begin{array}{l}\text { monofloral of } \\
\text { Mimosa } \\
\text { scabrella }\end{array}$} \\
\hline & bright & $\begin{array}{l}\text { Astrocaryum }(++), \\
\text { Mimosa scabrella }(++), \\
\text { Asteraceae }(++)\end{array}$ & heterofloral & & \\
\hline & dark & $\begin{array}{l}\text { immature pollen } \\
\text { grains, uredospores } \\
\text { and other pollen types }\end{array}$ & heterofloral & & \\
\hline
\end{tabular}


TABLE I (continuation)

\begin{tabular}{|c|c|c|c|c|c|}
\hline \multirow[b]{2}{*}{$\begin{array}{l}\text { Sample } \\
\text { register and } \\
\text { procedence }\end{array}$} & \multicolumn{3}{|c|}{ Technique 1} & \multicolumn{2}{|c|}{ Technique 2} \\
\hline & $\begin{array}{l}\text { Sub samples } \\
\text { established } \\
\text { by color of } \\
\text { pollen loads }\end{array}$ & $\begin{array}{l}\text { Pollen types } \\
\text { identified in the } \\
\text { sub samples }\end{array}$ & $\begin{array}{l}\text { Evaluation of } \\
\text { the sub samples }\end{array}$ & $\begin{array}{l}\text { Pollen types } \\
\text { identified in a pool } \\
\text { of } 2 \mathrm{~g} \text { of the sample }\end{array}$ & $\begin{array}{l}\text { Evaluation of } \\
\text { a pool of } 2 \mathrm{~g} \\
\text { of the sample }\end{array}$ \\
\hline 65-Bahia & green & $\begin{array}{l}\text { Borreria } \\
\text { densiflora }(++++)\end{array}$ & $\begin{array}{l}\text { monofloral of } \\
\text { Borreria } \\
\text { densiflora }\end{array}$ & & \\
\hline \multirow[t]{3}{*}{ 72-Espírito Santo } & $\begin{array}{l}\text { bright and } \\
\text { brown }\end{array}$ & $\begin{array}{l}\text { Euterpe edulis }(+++), \\
\text { Vernonia }(++)\end{array}$ & $\begin{array}{l}\text { bifloral of } \\
\text { Euterpe edulis } \\
\text { and Vernonia }\end{array}$ & \multirow{3}{*}{$\begin{array}{l}\text { Vernonia }(++) \\
\text { Cecropia }(++) \\
\text { Euterpe edulis }(+) \\
\text { Myrcia }(+) \\
\text { Eupatorium }(+) \\
\text { uredospores }(+)\end{array}$} & \multirow[t]{3}{*}{ heterofloral } \\
\hline & $\begin{array}{l}\text { orange and } \\
\text { yellow }\end{array}$ & $\begin{array}{l}\text { Persea }(+++) \\
\text { and several } \\
\text { pollen types }\end{array}$ & $\begin{array}{l}\text { heterofloral with } \\
\text { dominance of } \\
\text { Persea }\end{array}$ & & \\
\hline & $\begin{array}{l}\text { red and } \\
\text { violet }\end{array}$ & $\begin{array}{l}\text { without pollen } \\
\text { grains, only } \\
\text { uredospres }\end{array}$ & $\begin{array}{l}\text { without pollen } \\
\text { grains, only } \\
\text { uredospores }\end{array}$ & & \\
\hline \multirow[t]{5}{*}{ 74-Bahia } & yellow & Syagrus $(+++)$ & $\begin{array}{l}\text { without pollen } \\
\text { grains, only } \\
\text { uredospores }\end{array}$ & \multirow{5}{*}{$\begin{array}{l}\text { Cecropia }(++) \\
\text { Caesalpinia } \\
\text { peltophoroides }(++) \\
\text { Asteraceae }(++) \\
\text { Cyperus }(+) \\
\text { Syagrus }(+) \\
\text { Cocos nucifera }(+) \\
\text { Myrcia }(+)\end{array}$} & \multirow[t]{5}{*}{ heterofloral } \\
\hline & bright & $\begin{array}{l}\text { Cocos nucifera }(+++), \\
\text { Astrocaryum } \\
\text { aculeatissimum } \\
(+++)\end{array}$ & $\begin{array}{l}\text { bifloral of Cocos } \\
\text { nucifera and } \\
\text { Astrocaryum } \\
\text { aculeatissimum }\end{array}$ & & \\
\hline & dark & $\begin{array}{l}\text { Mangifera indica } \\
(++++)\end{array}$ & $\begin{array}{l}\text { without pollen } \\
\text { grains, only } \\
\text { uredospores } \\
\text { Mangifera indica }\end{array}$ & & \\
\hline & $\begin{array}{l}\text { orange and } \\
\text { green }\end{array}$ & $\begin{array}{l}\text { Borreria } \\
\text { verticillata }(+++), \\
\text { Bidens }(++)\end{array}$ & $\begin{array}{l}\text { bifloral of Borreria } \\
\text { verticillata and } \\
\text { Bidens }\end{array}$ & & \\
\hline & $\begin{array}{l}\text { brown and } \\
\text { orange }\end{array}$ & $\begin{array}{l}\text { Cocos nucifera }(+++), \\
\text { Cyperus }(+++)\end{array}$ & $\begin{array}{l}\text { bifloral of Cocos } \\
\text { nucifera and Cyperus }\end{array}$ & & \\
\hline \multirow[t]{2}{*}{ 77-Sergipe } & $\begin{array}{l}\text { bright and } \\
\text { orange }\end{array}$ & $\begin{array}{l}\text { Cocos }(++++), \\
\text { Montanoa }(+)\end{array}$ & $\begin{array}{l}\text { monofloral of } \\
\text { Cocos }\end{array}$ & \multirow{2}{*}{$\begin{array}{l}\text { Mimosa scabrella } \\
(+++) \\
\text { Cocos nucifera }(++) \\
\text { Eupatorium }(+)\end{array}$} & \multirow{2}{*}{$\begin{array}{l}\text { bifloral of } \\
\text { Mimosa } \\
\text { scabrella } \\
\text { (dominant) } \\
\text { and Cocos } \\
\text { nucifera } \\
\end{array}$} \\
\hline & brown & $\begin{array}{l}\text { Cocos nucifera } \\
(+++), \text { Mimosa } \\
\text { caesalpiniaefolia } \\
(+++)\end{array}$ & $\begin{array}{l}\text { bifloral of Cocos } \\
\text { nucifera and } \\
\text { Mimosa } \\
\text { caesalpiniaefolia }\end{array}$ & & \\
\hline \multirow[t]{3}{*}{ 80-Sergipe } & bright & $\begin{array}{l}\text { Cocos nucifera } \\
(++++), \\
\text { Richardia }(+)\end{array}$ & $\begin{array}{l}\text { monofloral of } \\
\text { Cocos nucifera }\end{array}$ & \multirow{3}{*}{$\begin{array}{l}\text { Mimosa scabrella } \\
(+++) \\
\text { Cocos nucifera }(++) \\
\text { Myrcia }(+)\end{array}$} & \multirow{3}{*}{$\begin{array}{l}\text { bifloral of } \\
\text { Mimosa } \\
\text { scabrella } \\
\text { (dominant) } \\
\text { and Cocos } \\
\text { nucifera }\end{array}$} \\
\hline & $\begin{array}{l}\text { dark and } \\
\text { caramel }\end{array}$ & $\begin{array}{l}\text { several pollen } \\
\text { types }\end{array}$ & heterofloral & & \\
\hline & orange & $\begin{array}{l}\text { Caesalpinia } \\
\text { peltophoroides } \\
(++++)\end{array}$ & $\begin{array}{l}\text { monofloral of } \\
\text { Caesalpinia } \\
\text { peltophoroides }\end{array}$ & & \\
\hline
\end{tabular}


TABLE I (continuation)

\begin{tabular}{|c|c|c|c|c|c|}
\hline \multirow[b]{2}{*}{$\begin{array}{l}\text { Sample } \\
\text { register and } \\
\text { procedence }\end{array}$} & \multicolumn{3}{|c|}{ Technique 1} & \multicolumn{2}{|c|}{ Technique 2} \\
\hline & $\begin{array}{l}\text { Sub samples } \\
\text { established } \\
\text { by color of } \\
\text { pollen loads }\end{array}$ & $\begin{array}{l}\text { Pollen types } \\
\text { identified in the } \\
\text { sub samples }\end{array}$ & $\begin{array}{l}\text { Evaluation of } \\
\text { the sub samples }\end{array}$ & $\begin{array}{l}\text { Pollen types } \\
\text { identified in a pool } \\
\text { of } 2 \mathrm{~g} \text { of the sample }\end{array}$ & $\begin{array}{l}\text { Evaluation of } \\
\text { a pool of } 2 \mathrm{~g} \\
\text { of the sample }\end{array}$ \\
\hline \multirow[t]{2}{*}{ 83-São Paulo } & $\begin{array}{l}\text { bright, green } \\
\text { and brown }\end{array}$ & $\begin{array}{l}\text { Schinus }(+++), \\
\text { Cecropia }(+++) \\
\text { and others }\end{array}$ & $\begin{array}{l}\text { bifloral of } \\
\text { Schinus and } \\
\text { Cecropia }\end{array}$ & \multirow{2}{*}{$\begin{array}{l}\text { Cecropia }(+++) \\
\text { Senecio }(+) \\
\text { Trema }(+) \\
\text { Myrcia }(+) \\
\text { Eupatorium }(+) \\
\text { Vernonia }(+)\end{array}$} & \multirow[t]{2}{*}{$\begin{array}{l}\text { monofloral of } \\
\text { Cecropia }\end{array}$} \\
\hline & orange & Senecio $(++++)$ & $\begin{array}{l}\text { monofloral of } \\
\text { Senecio }\end{array}$ & & \\
\hline \multirow[t]{3}{*}{ 84-Minas Gerais } & yellow & $\begin{array}{l}\text { Antigonon } \\
\text { leptopus }(++++)\end{array}$ & $\begin{array}{l}\text { monofloral of } \\
\text { Antigonon } \\
\text { leptopus }\end{array}$ & \multirow{3}{*}{$\begin{array}{l}\text { Antigonon leptopus } \\
(+++) \\
\text { Baccharis }(++) \\
\text { Mimosa scabrella } \\
(+), \text { Mimosa } \\
\text { caesalpiniaefolia } \\
(+), \text { unknown }(+)\end{array}$} & \multirow[t]{3}{*}{$\begin{array}{l}\text { monofloral of } \\
\text { Antigonon } \\
\text { leptopus }\end{array}$} \\
\hline & bright & $\begin{array}{l}\text { Baccharis }(+++) \\
\text { and others }\end{array}$ & $\begin{array}{l}\text { monofloral of } \\
\text { Baccharis }\end{array}$ & & \\
\hline & $\begin{array}{l}\text { orange and } \\
\text { brown }\end{array}$ & $\begin{array}{l}\text { several pollen } \\
\text { types }\end{array}$ & heterofloral & & \\
\hline \multirow[t]{3}{*}{ 85-Minas Gerais } & yellow & $\begin{array}{l}\text { Antigonon } \\
\text { leptopus }(+++), \\
\text { Cyperus }(+)\end{array}$ & $\begin{array}{l}\text { monofloral of } \\
\text { Antigonon } \\
\text { leptopus }\end{array}$ & \multirow[t]{3}{*}{$\begin{array}{l}\text { Cecropia }(+++) \\
\text { Myrcia }(++) \\
\text { Vernonia }(+)\end{array}$} & \multirow{3}{*}{$\begin{array}{l}\text { bifloral of } \\
\text { Cecropia } \\
\text { (dominant) } \\
\text { and Myrcia }\end{array}$} \\
\hline & $\begin{array}{l}\text { bright and } \\
\text { brown }\end{array}$ & $\begin{array}{l}\text { Anadenanthera }(++), \\
\text { Asteraceae }(++)\end{array}$ & heterofloral & & \\
\hline & dark & Myrcia $(++++)$ & $\begin{array}{l}\text { monofloral of } \\
\text { Myrcia }\end{array}$ & & \\
\hline \multirow[t]{3}{*}{ 86-São Paulo } & yellow & Eucalyptus $(++++)$ & $\begin{array}{l}\text { monofloral of } \\
\text { Eucalyptus }\end{array}$ & \multirow[t]{3}{*}{ Eucalyptus $(+++)$} & \multirow[t]{3}{*}{$\begin{array}{l}\text { monofloral of } \\
\text { Eucalyptus }\end{array}$} \\
\hline & orange & $\begin{array}{l}\text { Eucalyptus }(+++), \\
\text { Euphorbiaceae }(+++)\end{array}$ & $\begin{array}{l}\text { bifloral of } \\
\text { Eucalyptus and } \\
\text { Euphorbiaceae }\end{array}$ & & \\
\hline & brown & Eucalyptus $(++++)$ & $\begin{array}{l}\text { monofloral of } \\
\text { Eucalyptus }\end{array}$ & & \\
\hline \multirow[t]{2}{*}{ 87-Minas Gerais } & $\begin{array}{l}\text { bright and } \\
\text { brown }\end{array}$ & $\begin{array}{l}\text { Eucalyptus }(++), \\
\text { Vernonia }(++), \\
\text { Croton }(+)\end{array}$ & $\begin{array}{l}\text { bifloral of } \\
\text { Eucalyptus and } \\
\text { Vernonia }\end{array}$ & \multirow[t]{2}{*}{$\begin{array}{l}\text { Eucalyptus }(+++) \\
\text { Vernonia }(+)\end{array}$} & \multirow[t]{2}{*}{$\begin{array}{l}\text { monofloral of } \\
\text { Eucalyptus }\end{array}$} \\
\hline & $\begin{array}{l}\text { violet, orange } \\
\text { and yellow }\end{array}$ & $\begin{array}{l}\text { Eucalyptus }(+++), \\
\text { Senecio }(+++)\end{array}$ & $\begin{array}{l}\text { bifloral of } \\
\text { Eucalyptus and } \\
\text { Senecio }\end{array}$ & & \\
\hline \multirow[t]{3}{*}{ 88-São Paulo } & yellow & Baccharis $(++++)$ & $\begin{array}{l}\text { monofloral of } \\
\text { Baccharis }\end{array}$ & \multirow[t]{3}{*}{$\begin{array}{l}\text { Eucalyptus }(+++) \\
\text { Poaceae }(+)\end{array}$} & \multirow[t]{3}{*}{$\begin{array}{l}\text { monofloral of } \\
\text { Eucalyptus }\end{array}$} \\
\hline & bright & $\begin{array}{l}\text { Baccharis }(++), \\
\text { Syagrus }(++)\end{array}$ & $\begin{array}{l}\text { bifloral of } \\
\text { Baccharis and } \\
\text { Syagrus }\end{array}$ & & \\
\hline & brown & Eucalyptus $(++++)$ & $\begin{array}{l}\text { monofloral of } \\
\text { Eucalyptus }\end{array}$ & & \\
\hline \multirow[t]{2}{*}{ 97-Santa Catarina } & yellow & $\begin{array}{l}\text { Ilex }(++++), \\
\text { Onagraceae }(+)\end{array}$ & $\begin{array}{l}\text { monofloral of } \\
\text { Ilex }\end{array}$ & \multirow{2}{*}{$\begin{array}{l}\text { Eupatorium }(++), \\
\text { Melastomataceae } \\
(++), \text { Eucalyptus } \\
(++), \text { Vernonia }(+),\end{array}$} & \multirow[t]{2}{*}{ heterofloral } \\
\hline & bright & $\begin{array}{l}\text { Asteraceae }(+++) \\
\text { and others }\end{array}$ & $\begin{array}{l}\text { monofloral of } \\
\text { Asteraceae }\end{array}$ & & \\
\hline
\end{tabular}


TABLE I (continuation)

\begin{tabular}{|c|c|c|c|c|c|}
\hline \multirow[b]{2}{*}{$\begin{array}{l}\text { Sample } \\
\text { register and } \\
\text { procedence }\end{array}$} & \multicolumn{3}{|c|}{ Technique 1} & \multicolumn{2}{|c|}{ Technique 2} \\
\hline & $\begin{array}{l}\text { Sub samples } \\
\text { established } \\
\text { by color of } \\
\text { pollen loads }\end{array}$ & $\begin{array}{l}\text { Pollen types } \\
\text { identified in the } \\
\text { sub samples }\end{array}$ & $\begin{array}{l}\text { Evaluation of } \\
\text { the sub samples }\end{array}$ & $\begin{array}{l}\text { Pollen types } \\
\text { identified in a pool } \\
\text { of } 2 \mathrm{~g} \text { of the sample }\end{array}$ & $\begin{array}{l}\text { Evaluation of } \\
\text { a pool of } 2 \mathrm{~g} \\
\text { of the sample }\end{array}$ \\
\hline \multirow[t]{2}{*}{ 97-Santa Catarina } & orange & Senecio $(++++)$ & $\begin{array}{l}\text { monofloral of } \\
\text { Senecio }\end{array}$ & \multirow[t]{2}{*}{ Montanoa $(+)$} & \\
\hline & brown & Vernonia $(++++)$ & $\begin{array}{l}\text { monofloral of } \\
\text { Vernonia }\end{array}$ & & \\
\hline \multirow[t]{5}{*}{ 100-Paraná } & yellow & Alchornea $(+++)$ & $\begin{array}{l}\text { monofloral of } \\
\text { Alchornea }\end{array}$ & \multirow{5}{*}{$\begin{array}{l}\text { Sebastiania }(+++), \\
\text { Brassica }(++), \\
\text { Eucalyptus }(++)\end{array}$} & \multirow[t]{5}{*}{ heterofloral } \\
\hline & bright & $\begin{array}{l}\text { several pollen } \\
\text { types }\end{array}$ & heterofloral & & \\
\hline & $\begin{array}{l}\text { orange and } \\
\text { brown }\end{array}$ & $\begin{array}{l}\text { several pollen } \\
\text { types }\end{array}$ & heterofloral & & \\
\hline & green & Rosaceae $(++++)$ & $\begin{array}{l}\text { monofloral of } \\
\text { fruits (plum, } \\
\text { apple, pear) }\end{array}$ & & \\
\hline & $\begin{array}{l}\text { red and } \\
\text { brown }\end{array}$ & $\begin{array}{l}\text { Eucalyptus } \\
(++++) \text { and } \\
\text { uredosporos }\end{array}$ & $\begin{array}{l}\text { monofloral of } \\
\text { Eucalyptus and } \\
\text { uredospores }\end{array}$ & & \\
\hline \multirow[t]{4}{*}{ 111-Santa Catarina } & bright & $\begin{array}{l}\text { Eupatorium }(+++) \\
\text { Cocos nucifera }(++) \\
\text { Vernonia }(+)\end{array}$ & $\begin{array}{l}\text { bifloral of } \\
\text { Eupatorium and } \\
\text { Cocos nucifera }\end{array}$ & \multirow{4}{*}{$\begin{array}{l}\text { Vernonia }(++) \\
\text { Myrcia }(++) \\
\text { Montanoa }(++) \\
\text { Syagrus }(+) \\
\text { Poaceae }(+) \\
\text { Rubiaceae }(+) \\
\text { Crotalaria }(+)\end{array}$} & \multirow[t]{4}{*}{ heterofloral } \\
\hline & orange & Montanoa $(++++)$ & $\begin{array}{l}\text { monofloral of } \\
\text { Montanoa }\end{array}$ & & \\
\hline & brown & Vernonia $(++++)$ & $\begin{array}{l}\text { monofloral of } \\
\text { Vernonia }\end{array}$ & & \\
\hline & red & $\begin{array}{l}\text { Baccharis }(+++) \\
\text { Sebastiania }(++) \\
\text { Montanoa }(++)\end{array}$ & trifloral & & \\
\hline \multirow[t]{3}{*}{ 115-Sergipe } & bright & $\begin{array}{l}\text { Cocos nucifera } \\
(++++)\end{array}$ & $\begin{array}{l}\text { monofloral of } \\
\text { Cocos nucifera }\end{array}$ & \multirow{3}{*}{$\begin{array}{l}\text { Mimosa scabrella } \\
(+++), \text { Cocos } \\
\text { nucifera }(++)\end{array}$} & \multirow{3}{*}{$\begin{array}{l}\text { bifloral of } \\
\text { Mimosa } \\
\text { scabrella } \\
\text { (dominant) } \\
\text { and Cocos } \\
\text { nucifera }\end{array}$} \\
\hline & orange & $\begin{array}{l}\text { Mimosa scabrella } \\
(+++), \text { Asteraceae } \\
\text { (several pollen } \\
\text { types) }\end{array}$ & $\begin{array}{l}\text { monofloral of } \\
\text { Mimosa } \\
\text { scabrella }\end{array}$ & & \\
\hline & brown & $\begin{array}{l}\text { Mimosa scabrella } \\
(++++), \text { Cocos } \\
\text { nucifera }(++), \\
\text { Commelina }(++)\end{array}$ & $\begin{array}{l}\text { monofloral of } \\
\text { Mimosa } \\
\text { scabrella }\end{array}$ & & \\
\hline \multirow[t]{3}{*}{ 116-Sergipe } & yellow & Syagrus $(++++)$ & $\begin{array}{l}\text { monofloral of } \\
\text { Syagrus }\end{array}$ & \multirow{3}{*}{$\begin{array}{l}\text { Mimosa } \\
\text { caesalpiniaefolia } \\
(+++), \text { Cocos } \\
\text { nucifera }(++)\end{array}$} & \multirow{3}{*}{$\begin{array}{l}\text { bifloral of } \\
\text { Mimosa } \\
\text { caesalpiniaefolia } \\
\text { and Cocos } \\
\text { nucifera } \\
\text { (dominant) }\end{array}$} \\
\hline & bright & $\begin{array}{l}\text { Cocos nucifera } \\
(++++)\end{array}$ & $\begin{array}{l}\text { monofloral of } \\
\text { Cocos nucifera }\end{array}$ & & \\
\hline & orange & $\begin{array}{l}\text { several pollen } \\
\text { types }\end{array}$ & heterofloral & & \\
\hline
\end{tabular}


TABLE I (continuation)

\begin{tabular}{|c|c|c|c|c|c|}
\hline \multirow[b]{2}{*}{$\begin{array}{l}\text { Sample } \\
\text { register and } \\
\text { procedence }\end{array}$} & \multicolumn{3}{|c|}{ Technique 1} & \multicolumn{2}{|c|}{ Technique 2} \\
\hline & $\begin{array}{l}\text { Sub samples } \\
\text { established } \\
\text { by color of } \\
\text { pollen loads }\end{array}$ & $\begin{array}{l}\text { Pollen types } \\
\text { identified in the } \\
\text { sub samples }\end{array}$ & $\begin{array}{l}\text { Evaluation of } \\
\text { the sub samples }\end{array}$ & $\begin{array}{l}\text { Pollen types } \\
\text { identified in a pool } \\
\text { of } 2 \mathrm{~g} \text { of the sample }\end{array}$ & $\begin{array}{l}\text { Evaluation of } \\
\text { a pool of } 2 \mathrm{~g} \\
\text { of the sample }\end{array}$ \\
\hline 116-Sergipe & brown & $\begin{array}{l}\text { Mimosa } \\
\text { caesalpiniaefolia } \\
(++++), \\
\text { Mimosaceae } \mathrm{Mv}(++)\end{array}$ & $\begin{array}{l}\text { monofloral of } \\
\text { Mimosa } \\
\text { caesalpiniaefolia }\end{array}$ & & \\
\hline \multirow[t]{3}{*}{ 144-Bahia } & yellow & $\begin{array}{l}\text { Syagrus }(+++), \\
\text { Cocos nucifera }(+), \\
\text { Eupatorium }(+)\end{array}$ & $\begin{array}{l}\text { monofloral of } \\
\text { Syagrus }\end{array}$ & \multirow[t]{3}{*}{$\begin{array}{l}\text { Baccharis }(+++), \\
\text { Cocos nucifera }(++) \\
\text { Syagrus }(+)\end{array}$} & \multirow[t]{3}{*}{ heterofloral } \\
\hline & bright & $\begin{array}{l}\text { Cocos nucifera } \\
(++++)\end{array}$ & $\begin{array}{l}\text { monofloral of } \\
\text { Cocos nucifera }\end{array}$ & & \\
\hline & several colors & $\begin{array}{l}\text { several pollen } \\
\text { types }\end{array}$ & heterofloral & & \\
\hline \multirow[t]{3}{*}{ 145-Bahia } & caramel & $\begin{array}{l}\text { Cocos nucifera } \\
(+++) \text {, Poaceae } \\
(+++)\end{array}$ & $\begin{array}{l}\text { bifloral of Cocos } \\
\text { nucifera and } \\
\text { Poaceae }\end{array}$ & \multirow{3}{*}{$\begin{array}{l}\text { Mimosa scabrella } \\
(++), \text { Schinus }(++), \\
\text { Poaceae }(+), \\
\text { Cocos nucifera }(+), \\
\text { Mimosa } \\
\text { caesalpiniaefolia } \\
(+), \text { Eupatorium }(+) \\
\text { Syagrus }(+)\end{array}$} & \multirow[t]{3}{*}{ heterofloral } \\
\hline & bright & $\begin{array}{l}\text { Cocos nucifera } \\
(+++), \\
\text { Astrocaryum } \\
\text { aculeatissimum } \\
(+++)\end{array}$ & $\begin{array}{l}\text { bifloral of Cocos } \\
\text { nucifera and } \\
\text { Astrocaryum } \\
\text { aculeatissimum }\end{array}$ & & \\
\hline & several colors & $\begin{array}{l}\text { Mimosa } \\
\text { caesalpiniaefolia } \\
(++), \text { Trema }(++), \\
\text { and others }\end{array}$ & heterofloral & & \\
\hline \multirow[t]{4}{*}{ 146-Bahia } & caramel & $\begin{array}{l}\text { Poaceae }(++++) \\
\text { Triumfetta }(+)\end{array}$ & $\begin{array}{l}\text { monofloral of } \\
\text { Poaceae }\end{array}$ & \multirow{4}{*}{$\begin{array}{l}\text { Cocos nucifera }(++) \\
\text { Schinus }(++) \\
\text { Poaceae }(++) \\
\text { Mimosa } \\
\text { caesalpiniaefolia }(++) \\
\text { Mimosa scabrella }(+)\end{array}$} & \multirow[t]{4}{*}{ heterofloral } \\
\hline & bright & $\begin{array}{l}\text { Cocos nucifera } \\
(++++)\end{array}$ & $\begin{array}{l}\text { monofloral of } \\
\text { Cocos }\end{array}$ & & \\
\hline & $\begin{array}{l}\text { dark and } \\
\text { yellow }\end{array}$ & $\begin{array}{l}\text { several pollen } \\
\text { types }\end{array}$ & heterofloral & & \\
\hline & orange & $\begin{array}{l}\text { Portulaca }(+++) \\
\text { Commelina }(++)\end{array}$ & $\begin{array}{l}\text { bifloral of } \\
\text { Portulaca and } \\
\text { Commelina }\end{array}$ & & \\
\hline \multirow[t]{5}{*}{ 150-Espírito Santo } & yellow & $\begin{array}{l}\text { Alchornea }(+++) \text {, } \\
\text { Arecaceae }(+) \\
\text { and others }\end{array}$ & $\begin{array}{l}\text { monofloral of } \\
\text { Alchornea }\end{array}$ & \multirow{5}{*}{$\begin{array}{l}\text { Eucalyptus }(++) \\
\text { Cecropia }(++) \\
\text { Myrcia }(++) \\
\text { Asteraceae }(+) \\
\text { Cocos nucifera }(+) \\
\text { Poaceae }(+) \\
\text { Alchornea }(+)\end{array}$} & \multirow[t]{5}{*}{ heterofloral } \\
\hline & caramel & $\begin{array}{l}\text { Poaceae }(+++), \\
\text { Croton }(+)\end{array}$ & $\begin{array}{l}\text { monofloral of } \\
\text { Poaceae }\end{array}$ & & \\
\hline & $\begin{array}{l}\text { bright and } \\
\text { brown }\end{array}$ & $\begin{array}{l}\text { Cocos nucifera } \\
(+++), \text { Syagrus }\end{array}$ & $\begin{array}{l}\text { bifloral of Cocos } \\
\text { nucifera and }\end{array}$ & & \\
\hline & dark and red & $\begin{array}{l}\text { several pollen } \\
\text { types }\end{array}$ & heterofloral & & \\
\hline & $\begin{array}{l}\text { orange and } \\
\text { green }\end{array}$ & $\begin{array}{l}\text { Montanoa } \\
(++++), \\
\text { Asteraceae }(+)\end{array}$ & $\begin{array}{l}\text { monofloral of } \\
\text { Montanoa }\end{array}$ & & \\
\hline
\end{tabular}


Preparing slides in five folds, the same pollen spectrum was obtained.

Detailed discussion of the data obtained by the use of the two techniques of pollen load batches analysis (Table I).

Pollen grain color of a plant taxon (third column in Table I) can change from bright to dark as it may be observed in Cocos (samples 55, 74, 77, 115, 145, 146), Vernonia (samples 58, 111), Eucalyptus (samples 86, 87) and Baccharis (sample 88) pollen grains, and in the Mimosa scabrella pollen type (sample 115). Time to air exposition of pollen grains resulting in exine and cytoplasm oxidation could be responsible for different colored pollen loads of a plant species.

Using technique 1, all pollen batches were heterofloral. Technique 2 revealed monofloral (samples 56, 57, $65,83,84,86,87,88$ ) and bifloral (samples 55, 77, 80, $85,115,116)$ samples besides the heterofloral ones.

The monofloral samples presented five dominant pollen types: Cocos nucifera and Mimosa scabrella (= M. sensitiva) pollen types from the state of Bahia, Cecropia and Eucalyptus from São Paulo State, Antigonon leptopus (a garden species) and Eucalyptus from the state of Minas Gerais. These commercial batches have obtained the best evaluation.

The bifloral samples presented six important pollen types: Cocos nucifera and Cyperus from the state of Piaui, Mimosa scabrella and Cocos nucifera in three samples from the state of Sergipe, Mimosa caesalpiniaefolia and Cocos nucifera also from the state of Sergipe, and Cecropia and Myrcia from the state of Minas Gerais. These commercial batches may receive a good evaluation.

The heterofloral pollen load batches analyzed by techniques 1 and 2 do not have palynological definition of any dominance. Their botanical origin is variable depending upon several factors and their reproduction must not be effective.

A curious composition of some pollen loads was made of uredospores of fungi detected by technique 1 (in samples 59, 72, 74, 100). Uredospores never appeared using technique 2. As so, their contribution to any of the pollen batches was not significant. Cladosporium sp. spores were also collected by bees in the state of Minas Gerais during an alimentary scarcity (Modro et al. 2009b).
In conclusion, using technique 1 based upon color analysis, more pollen types were identified, but no dominance of pollen type or plant species in a commercial pollen batch was reported. When using the technique 2, besides the monofloral batches, more bifloral and less heterofloral batches were recognized. This result shows that a better characterization of a large pollen load batch composition of commercial interest was obtained when using the last technique.

\section{RESUMO}

É exigida alta qualidade para a comercialização de pólen apícola. A fim de atender o consumidor com a melhor identificação da origem botânica e floral do produto, 25 partidas de pólen apícola foram investigadas usando duas diferentes técnicas na preparação dos grãos de pólen. A primeira partiu da identificação das cargas polínicas contidas em dois gramas de cada partida bem misturada segundo suas cores. A segunda visava identificar os grãos de pólen de um agrupamento ("pool") de todas as cargas polínicas contidas em dois gramas de cada amostra. O melhor resultado foi obtido pela última técnica, quando uma suspensão de grãos de pólen era gotejada sobre uma lâmina de microscopia e cerca de 500 grãos de pólen eram contados por amostra. Esta análise resultou no reconhecimento de partidas monoflorais e biflorais de pólen apícola, enquanto que usando a primeira técnica, todas as amostras receberam a diagnose heterofloral.

Palavras-chave: Apis, cargas de pólen, partidas comerciais de pólen, análise polínica, origem botânica.

\section{REFERENCES}

Almeida-Muradian LB, Pamplona LC, Coimbra S AND BARTH OM. 2005. Chemical composition and botanical evaluation of dried bee pollen pellets. J Food compos anal 18: 105-111.

BARTH OM. 1989. O pólen no mel brasileiro. Editora Luxor, $151 \mathrm{p}$.

BARTH OM, Munhoz MC AND Luz CFP. 2009. Botanical origin of Apis pollen loads using color, weight and pollen morphology data. Acta aliment 38: 133-139. DOI: 10.1556/AAlim.2008.0026.

Carvalho CAL and Marchini LC. 1999. Tipos polínicos coletados por Nannotrigona testaceicornis e Tetragonisca angustula (Hymenoptera, Apidae, Meliponinae). Sci Agri (Piracicaba, Braz.) 56: 717-722. 
Louveaux J, Maurizio A and Vorwohl G. 1978. Methods of melissopalynology. Bee World 59: 139-157.

Marques-Souza AC, Miranda IPA, Moura CO, RaBELO A AND BARBosA EM. 2002. Características morfológicas e bioquímicas do pólen coletado por cinco espécies de Meliponíneos da Amazônia Central. Acta Amaz 32: 217-229.

Modro AFH, Message D, Luz CFP And Meira-Neto JAA. 2007. Composição e qualidade de pólen apícola coletado em Minas Gerais. Pesqui agropecu bras 42: 10571065.

Modro AFH, Silva IC, Luz CFP And Message D. 2009a. Analysis of pollen load based on color, physicochemical composition and botanical source. An Acad Bras Cienc 81: 281-285.
Modro AFH, Silva IC, Message D And Luz CFP. 2009b. Saprophytic fungus collection by Africanized bees in Brazil. Neotrop Entomol 38(3): 434-436.

Noor MJ, KAHn MA ANd CAMPhor ES. 2009. Palynological analysis of pollen loads from pollen sources of honeybees in Islamabad, Pakistan. Pak J Bot 41: 495-501.

Novais JS, Lima LCL AND SANTOS FAR. 2009. Botanical affinity of pollen harvested by Apis mellifera L. in a semiarid area from Bahia, Brazil. Grana 48: 224-234.

Roubik DW And Moreno JE. 1991. Pollen and spores of Barro Colorado Island. Missouri Botanical Garden, Monographs in Systematic Botany, 263 p. 